\title{
Ensayo de método para el diseño de dispositivos de sombra en espacios públicos del trópico. Caso Medellín
}

\author{
Lucas Arango Díaz \\ Juliana Sánchez Jaramillo** \\ Esteban Tejada Urrego***
}

Artículo

\begin{abstract}
Afiliaciónes:
*Oficina de arquitectura: La B arquitectura + Bioclimática.

**Universidad Nacional de Colombia, sede Medellín.

***Universidad de San Buenaventura, Medellín. Colombia.
\end{abstract}

E-mail: lucas.arango.diaz@gmail.com jsanchezja.19@gmail.com etejadau88@gmail.com

Recibido: 20 de agosto del 2019

\section{Lucas Arango Díaz}

Arquitecto Universidad Nacional de Colombia Magister en Arquitectura y Urbanismo Universidade federal de Santa Catarina, Brasil. Gerente General LaB S.A.S. Docente ocasional Universidad Nacional de Colombia. Docente investigador medio tiempo Universidad de San Buenaventura, Medellín.

\section{Juliana Sánchez Jaramillo}

Arquitecta Universidad Nacional de Colombia, Sede Medellín (Colombia). Magister en Bioclimática (en proceso) Universidad de San Buenaventura Medellín. Asesora Bioclimática LaB S.A.S.

\section{Esteban Tejada Urrego}

Arquitecto Institución Universitaria Colegio Mayor de Antioquia, Medellín (Colombia). Asesor Bioclimático LaB S.A.S.

\section{Resumen:}

En Colombia, la inclusión de estrategias bioclimáticas en el proceso proyectual no suele realizarse desde las fases preliminares de diseño, sino en las fases medias o finales, cuando pocos cambios pueden realizarse debido al nivel de avance del proyecto. Este artículo resalta la importancia de considerar herramientas o métodos útiles para tomar decisiones a partir de criterios bioclimáticos desde las fases iniciales del proceso proyectual, de manera que la bioclimática se convierta en una premisa proyectual que incide en la imagen y funcionalidad. En este artículo se explica un método para la inclusión de elementos de control solar de una cubierta sobre una plaza localizada en la ciudad de Medellín, Colombia (Latitud: 6¹5'6.62" N, Longitud: 75³3'48.92" O, Altitud: 1500 m.s.n.m), como parte del estudio bioclimático del proyecto Bazar de los Puentes. El proyecto, diseñado por el Arquitecto Sebastián Monsalve Gómez para la Empresa de Desarrollo Urbano, EDU, hace parte del plan para la recuperación estratégica de varios sectores urbanos de la ciudad. El método permitió que el grupo de arquitectos configurara el sistema de cubiertas de acuerdo con la conveniencia de sombra.

Palabras clave: arquitectura; espacio público; bioclimática; control solar; método de diseño.

Method test for the design of shade mechanisms on public space roofs in the tropics. A case study in Medellín

\footnotetext{
Abstract:

In Colombia, the inclusion of bioclimatic strategies in the architectural design process is not usually done in earlier design phases but in the middle or final phases, when few changes can be made due to the progress of the Project. This paper highlights the importance of considering useful tools or methods to take architectural decisions based on bioclimatic criteria from earliest phases of the project process so that bioclimatic design becomes a project premise and a reason that affects the image and functionality. This paper explains a method for the inclusion of solar control elements of a roof over a plaza located in the city of Medellín, Colombia (Latitude: 6¹5'6.62" N, Longitude: $75^{\circ} 33^{\prime} 48.92^{\prime \prime}$ O, Altitude: 1500 m.a.s.I) as part of the Bioclimatic Studies, a project known as Bazar de los Puentes. The project, designed by the Architect Sebastián Monsalve Gómez for the Urban Development Company (EDU in Spanish), is part of the plan for the strategic recovery of several urban sectors in the city. The method allowed the group of architects to configure the roofing system according to the shade convenience.
} 


\section{Introducción}

I confort térmico en espacios urbanos ha sido ampliamente estudiado por varios investigadores desde el punto de vista de los aspectos físicos (Boukhelkhal \& Bourbia, 2016; Wang, Ni, Peng \& Xia, 2018; Xu, Hong, Jiang, An \& Zhang, 2019) y de la adaptación de las personas (Ahmed, 2003; Cheng, Gou, Zhang, Feng \& Huang, 2019; Kicovic, Vuckovic, Markovic \& Jovic, 2019; Nikolopoulou \& Steemers, 2003; Zabetian \& Kheyroddin, 2019). Varios estudios se han enfocado en identificar las principales estrategias bioclimáticas que, en términos generales, favorecerían el desempeño bioclimático en espacios exteriores (Lai, Liu, Gan, Liu \& Chen, 2019).

En términos generales, el diseño bioclimático de espacios públicos en localidades intertropicales con clima cálido 0 templado se enfoca en la provisión de sombra abundante (Ahmed, 2003; Mazhar, Brown, Kenny \& Lenzholzer, 2015; Xu et al., 2019), el aprovechamiento de las corrientes de viento y la protección frente a la lluvia. Entre los hallazgos que encontró Ahmed (2003) en una investigación de espacios urbanos específicamente enfocada en los trópicos, se destaca que la sombra en espacios urbanos entre las 10:30 am y las 3:30 pm resulta altamente deseable. Yang, Wong y Jusuf (2013), al confirmar que el factor que más influye en la sensación de confort en espacios exteriores en la exposición a la radiación solar directa, otorgan gran importancia a los dispositivos de control solar en espacios exteriores. Ouali, El Harrouni, Abidi y Diab (2020) resaltan el papel de la sombra, entre otras variables, sobre la temperatura media radiante, la cual relacionan directamente con la posibilidad de generación de confort térmico en espacios exteriores. Boukhelkhal \& Bourbia (2016) evidenciaron la importancia de la relación de la geometría de los espacios construidos y la vegetación en la generación de sombra para el mejoramiento del confort térmico en espacios exteriores y de microclimas agradables. Waldron (2017) señala en su estudio que factores como la iluminancia, la velocidad del viento y la temperatura radiante media en la sombra influyen en el comportamiento social de las personas, mientras que la humedad relativa y la temperatura radiante media en el sol afectan los comportamientos individuales. Lin, Matzarakis y Hwang (2010) encontraron que las ubicaciones poco sombreadas eran incómodas en verano y las ubicaciones altamente sombreadas eran incómodas en invierno. Los niveles medios de sombreado contribuyeron al período de confort térmico más largo en todo un año, mientras que los espacios con poca o excesiva sombra tienen períodos cortos de confort térmico.

En Medellín, Colombia (Latitud: 6¹5'6.62" N, Longitud: 75³3'48.92" O, Altitud: 1500 m.s.n.m en el centro), que posee un clima Tropical Monzónico, Am según la clasificación climática de Köppen-Geiger y donde la temperatura del aire al medio día puede alcanzar $\operatorname{los} 31^{\circ} \mathrm{C}$, la sombra en zonas de estancia o de recorridos exteriores resulta la estrategia más útil para favorecer la comodidad térmica en espacios urbanos. Para este fin, son utilizadas diversas posibilidades de sistemas de control solar, cuya eficiencia se mide según su capacidad de obstruir los rayos solares cuando estos resultan inconvenientes desde el punto de vista térmico. Varios métodos para el dimensionamiento de sistemas de control solar han sido desarrollados (Aroztegui, 1980; Dubois, 2000; Dutra, Lamberts, Oscar \& Pereira, n.d.; Kaftan \& Marsh, 2005; Kaftan, 2001). Los métodos geométricos donde se aplican diagramas solares y máscaras de sombra y se considera la deseabilidad de sombra para cada mes del año y hora del día son útiles para identificar los ángulos solares críticos para el dimensionamiento de los dispositivos de sombra (Bittencourt, 1988; Mesa \& Morillón, 2005; Morillón Gálvez \& Mejía Domínguez, 2004). También se utilizan programas computacionales en donde a partir de un modelo tridimensional de los elementos de control solar es posible visualizar las Horas de Sombra Diaria (HSD), es decir, la distribución de la sombra proyectada por el elemento sólido en un plano de análisis determinado, tanto en espacio como en tiempo (García \& Salazar, 2006; Salazar, 2007; Salazar Trujillo, 2009; Waldron \& Salazar, 2013). En algunos casos, los métodos geométricos se complementan con la aplicación de programas computacionales para verificar el desempeño de los dispositivo (González \& Martínez, 2014). Asimismo, están los métodos de cálculo basados en ecuaciones matemáticas, como es el caso de la implementada por Salazar Trujillo (2014) para calcular la penumbra o dispersión de la sombra. Otro método es a través de maquetas o modelos a escala que son llevadas al 
Figura 1a. Proyecto Bazar de los Puentes. Imagen generada por el Arq. Sebastián

Monsalve Gómez para la Empresa de Desarrollo Urbano (EDU).

Fuente: Sebastián Monsalve Gómez. sitio para recrear las condiciones reales de orientación y entorno inmediato, en donde se puede visualizar la incidencia solar y el desempeño de los elementos de control solar (Salazar Trujillo, García Cardona \& Gónzalez Castaño, 2006; Villalba, Monteoliva \& Pattini, 2011)

A pesar de esto, al menos en todo el territorio colombiano y muy posiblemente en gran parte del territorio latinoamericano, el planteamiento $y / 0$ desarrollo de estrategias de sombreamiento se lleva a cabo a partir de métodos tipo ensayo-error y en las fases medias o finales del proceso proyectual cuando las herramientas de simulación sirven para validar resultados y cuando pocas modificaciones pueden ser realizadas (Haghparast \& Marsh, 2004; Marsh \& Carruthers, 1995; Marsh, 1996). Estos autores plantean la necesidad de crear mecanismos que permitan generar datos útiles sobre el desempeño bioclimático desde las fases iniciales del proyecto, cuando los modelos arquitectónicos están incompletos 0 apenas en proceso. Varios autores han reportado con éxito la aplicación de estas metodologías desde las fases iniciales del proyecto, es decir, la inclusión de la bioclimática como premisa proyectual (Arango-Díaz, Giraldo Vásquez, Rupp, Sierra \& Gallego, 2015; Naranjo et al., 2015; Salazar, García, González, Arango-Díaz \& Giraldo, 2009; Torres-Gómez et al., 2015).

Este artículo describe una metodología de diseño de elementos de control solar para una cubierta de un proyecto de diseño urbano localizada en Medellín, Colombia. El proyecto, denominado Bazar de los Puentes y diseñado por el Arquitecto Sebastián Monsalve Gómez por pedido de la Empresa de Desarrollo Urbano (EDU), consiste en la readecuación de una zona urbana de alrededor de $8000 \mathrm{~m}^{2}$ de tal manera que posea cualidades estéticas y bioclimáticas que favorezcan el uso y la apropiación por parte de los ciudadanos (ver Figura 1) tal como llama la atención de Lai et al. (2019). En la readecuación, se optó por incluir una gran cubierta con dispositivos para generar sombra suficiente para los puestos comerciales que se dispondrían en el espacio público. Asimismo, se procuró evitar la disposición de cerramientos verticales opacos para permitir un control visual del espacio y el libre paso de transeúntes, complementando el proyecto con algunos jardines de especies vegetales bajas como apoyo al control solar de la cubierta para los ángulos más inclinados.

El objetivo de esta metodología consistió en dotar a los arquitectos diseñadores de una herramienta o estrategia metodológica que les permitiera especificar las cubiertas para alcanzar el lenguaje deseado a partir de criterios de sombra desde las fases iniciales del proyecto. Así, estos no esperarían a que se realizara una propuesta de cubierta para que, por ensayo error, se afinara.

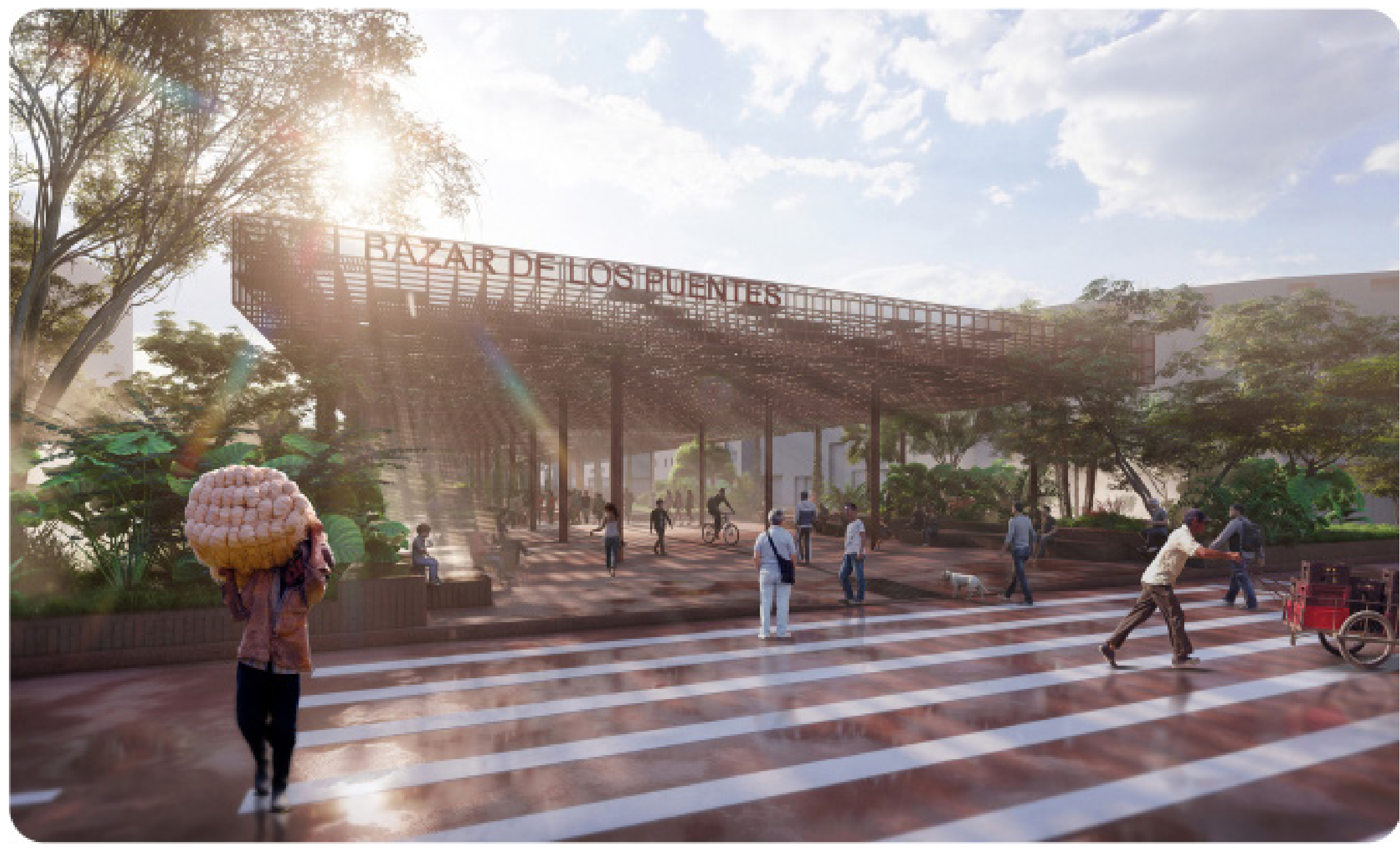



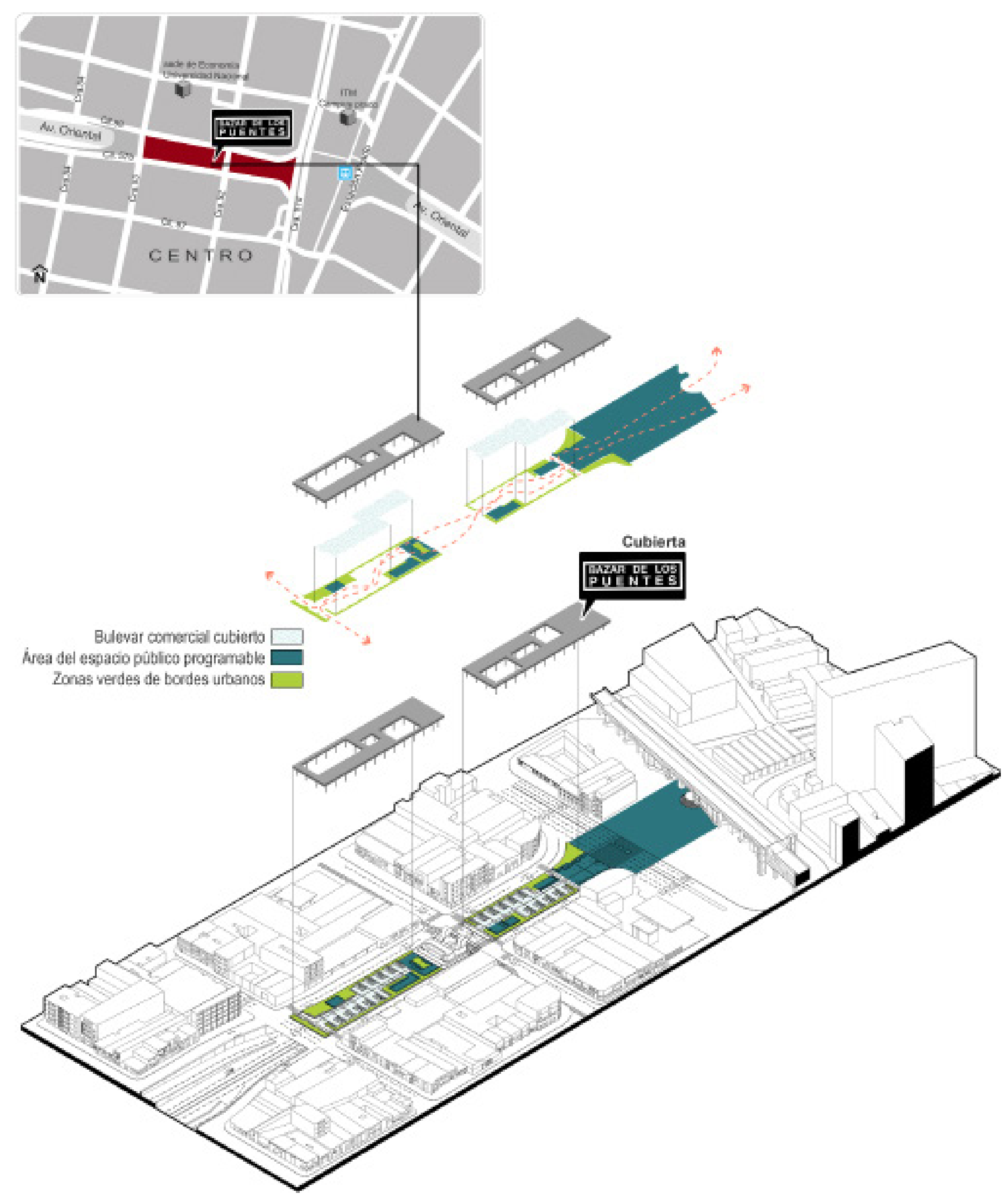

Figura 1b. Proyecto Bazar de los Puentes.

Imagen generada por el Arq. Sebastián

Monsalve Gómez para la Empresa de Desarrollo

Urbano (EDU).

Fuente: Sebastián Monsalve Gómez. 


\section{Características de la cubierta}

Durante el proceso de la asesoría bioclimática, el grupo de arquitectos diseñadores planteó el diseño de una cubierta tipo malla espacial con módulos de $0,5 \mathrm{~m} \times 0,5 \mathrm{~m}$, altura total de $1 \mathrm{~m}$ en el centro y $1,5 \mathrm{~m}$ en los bordes. Toda la malla se dispuso a $5 \mathrm{~m}$ del piso (ver Figura 2). A partir de esta estructura, el grupo de arquitectos diseñadores planteó disponer paneles horizontales de $0,5 \mathrm{~m} \times 0,5 \mathrm{~m}$ en los módulos de la malla espacial con una distribución aparentemente aleatoria, pero que cumpliera con criterios de control solar.

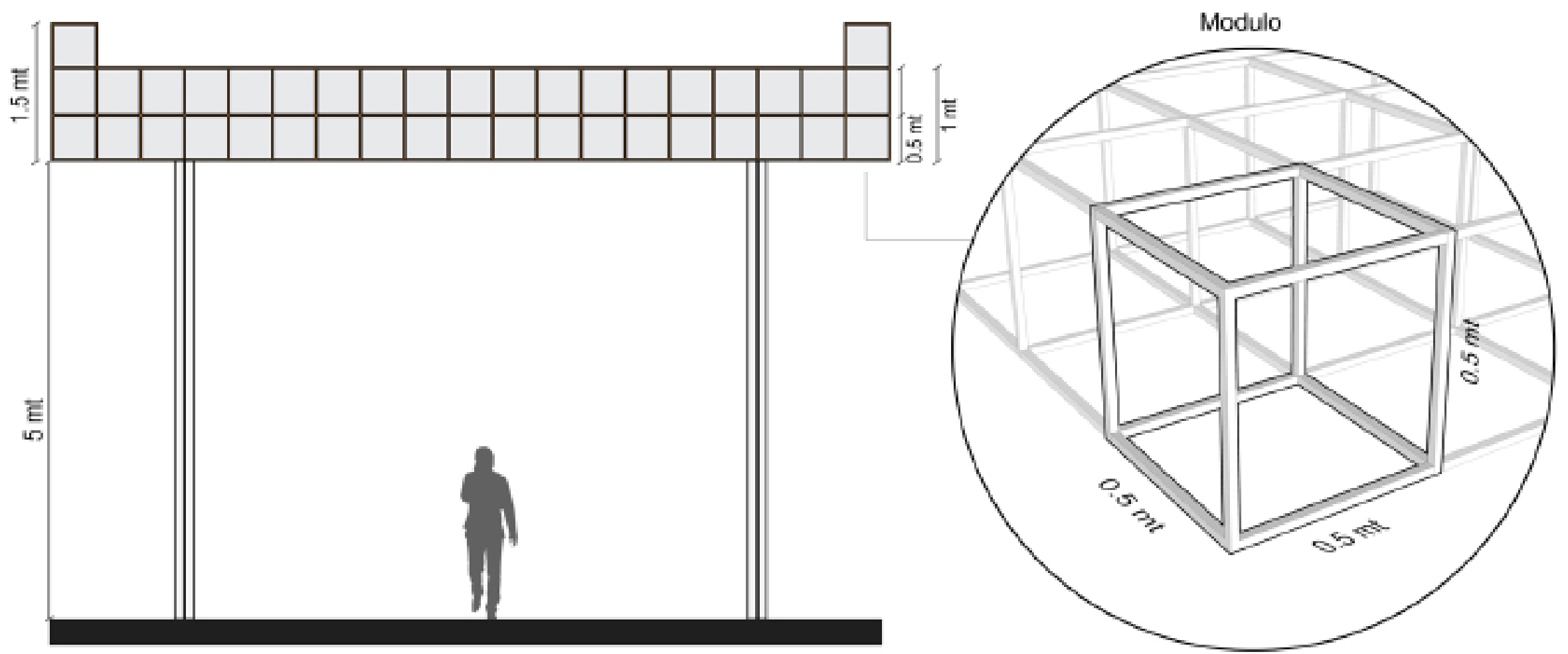

Figura 2. Planteamiento malla espacial para la generación de cubierta.

Fuente: elaboración propia.

Figura 3. Cuadricula para generación de la herramienta.

Fuente: elaboración propia.

\section{Diseño de la herramienta}

Con el objetivo de apoyar al equipo de arquitectos diseñadores en la disposición de paneles horizontales sobre la malla espacial en las diferentes alturas, de manera que pareciera una distribución aleatoria, pero que garantizara sombra de acuerdo con criterios bioclimáticos, fue diseñada una herramienta haciendo uso de una cuadrícula de $0,5 \mathrm{~m} \times 0,5 \mathrm{~m}$ y un punto a sombrear. Para facilitar la lectura del gráfico, tanto en el eje "x" como en el eje "y", cada columna y cada fila agrupó un par de celdas. Las columnas se nombraron con letras y las filas con números. En el centro de cuadrícula, es decir, la intersección entre la columna "0" y la fila "0", se enmarca el punto en el espacio que se desearía proteger del sol. La Figura 3 muestra la malla anteriormente descrita.

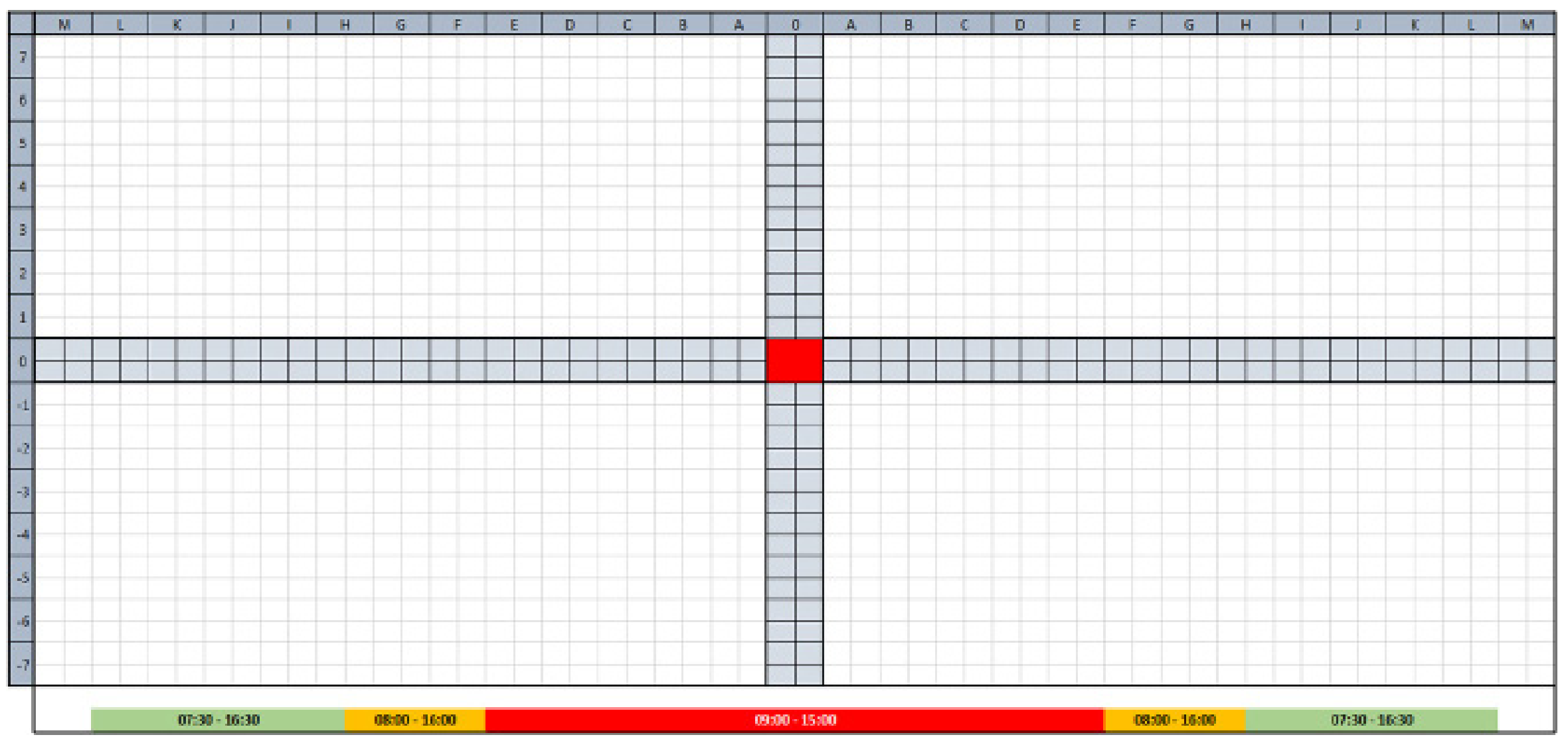


A continuación, asumiendo que la malla se encontraba a $5 \mathrm{~m}$ de altura del piso, fueron resaltadas las celdas con colores según el criterio de control solar. Por ejemplo, en la Figura 4, el color rojo resalta las celdas que en caso de poseer panel horizontal de protección solar a la altura de $5 \mathrm{~m}$, garantizarían sombra suficiente entre las 9:00h y las 15:00h. Si a las rojas se les suma las celdas amarillas, se alcanzaría a obstruir el sol entre las 8:00h y las 16:00h. Ya considerando las verdes, amarillas y rojas, se garantizaría sombra entre las 7:30 y las 16:30h sobre el punto medio.

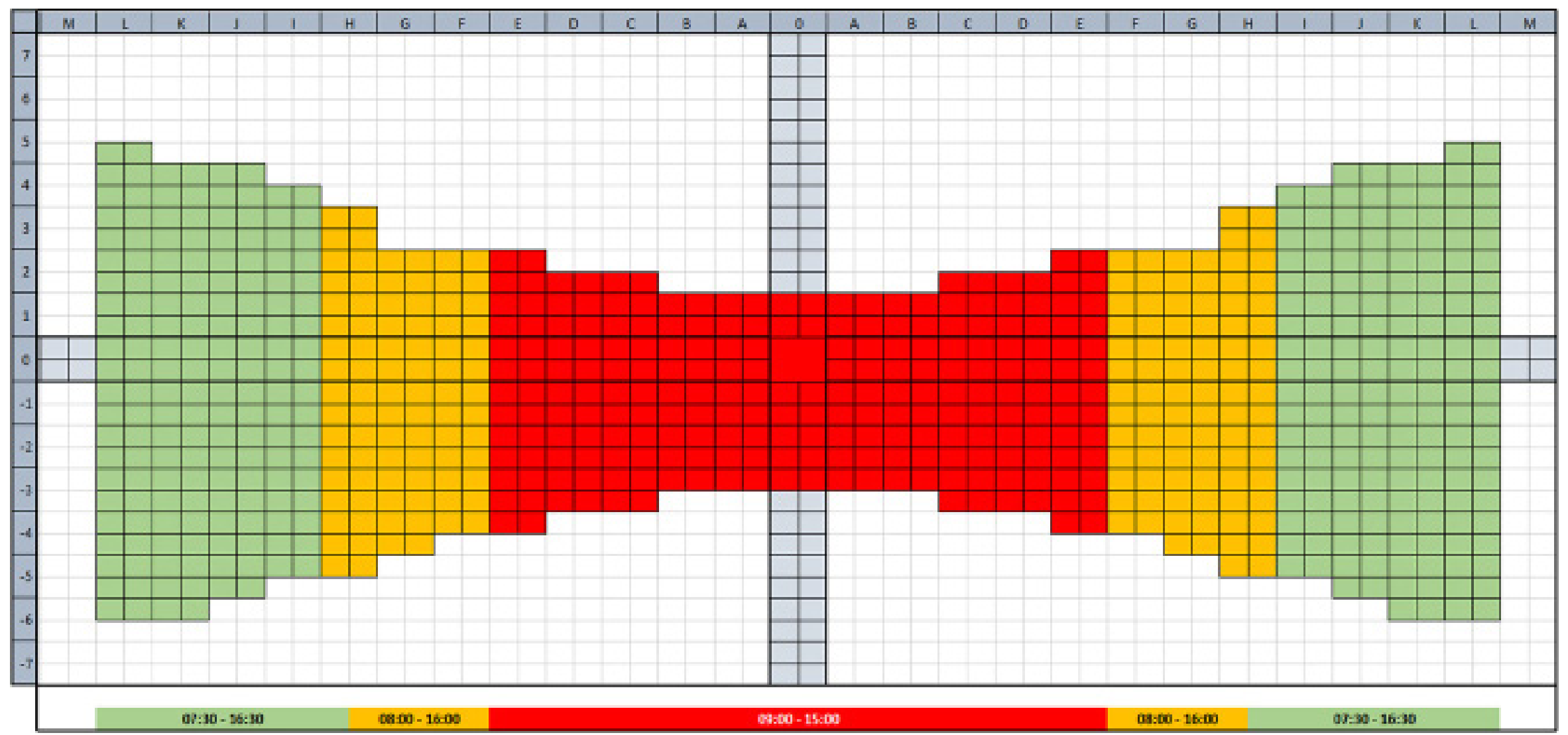

Figura 4. Sombreamiento ideal - Altura 5.0m. Fuente: elaboración propia.

No obstante, debido a que entre las premisas de diseño se encontraba aparentar aleatoriedad entre la disposición de paneles horizontales sobre la malla espacial, fueron generadas las Figuras 5 y 6 . La Figura 5 muestra a una altura de $5,5 \mathrm{~m}$, como deberían desplazarse los paneles previamente calculados en la Figura 4, de manera que garantizaran la misma sombra que se lograría con esos paneles en la cubierta a $5 \mathrm{~m}$. Por ejemplo, en la Figura 5 se muestra que los paneles D1 deben desplazarse 1 celda hacia el oeste a 5,5m de altura para garantizar la misma sombra que se lograría en esta celda a $5 \mathrm{~m}$ de altura o que la celda 13 debería desplazarse a $5,5 \mathrm{~m}$ de altura, 2 celdas hacia el sur y 2 celdas hacia el este para garantizar la misma sombra que lograría con esta celda a $5 \mathrm{~m}$ de altura.

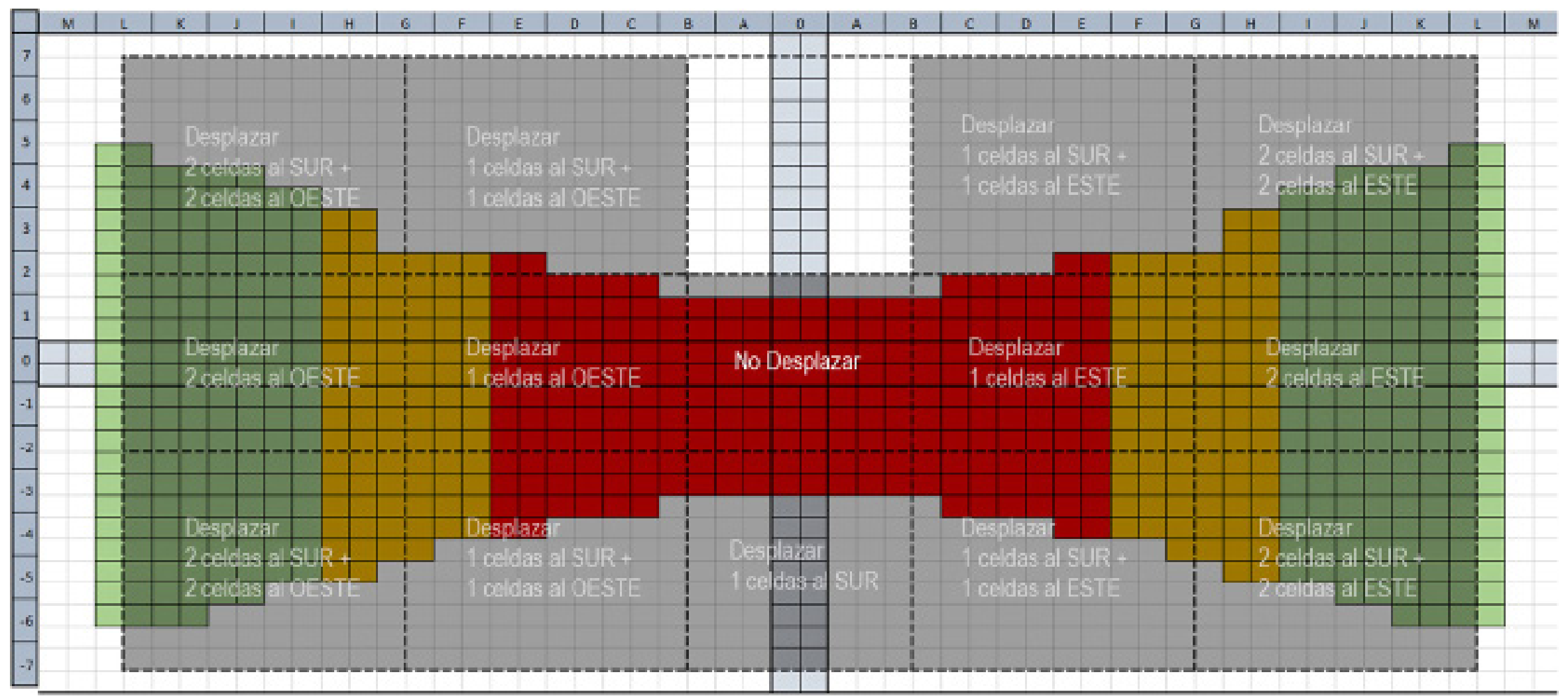

Figura 5. Sombreamiento ideal - Altura 5.5m. Fuente: elaboración propia.
Del mismo modo, la Figura 14 muestra los desplazamientos que deben realizarse a $6 \mathrm{~m}$ con relación a las celdas resaltadas a $5 \mathrm{~m}$ de altura, de manera que se garantice la sombra deseada. 


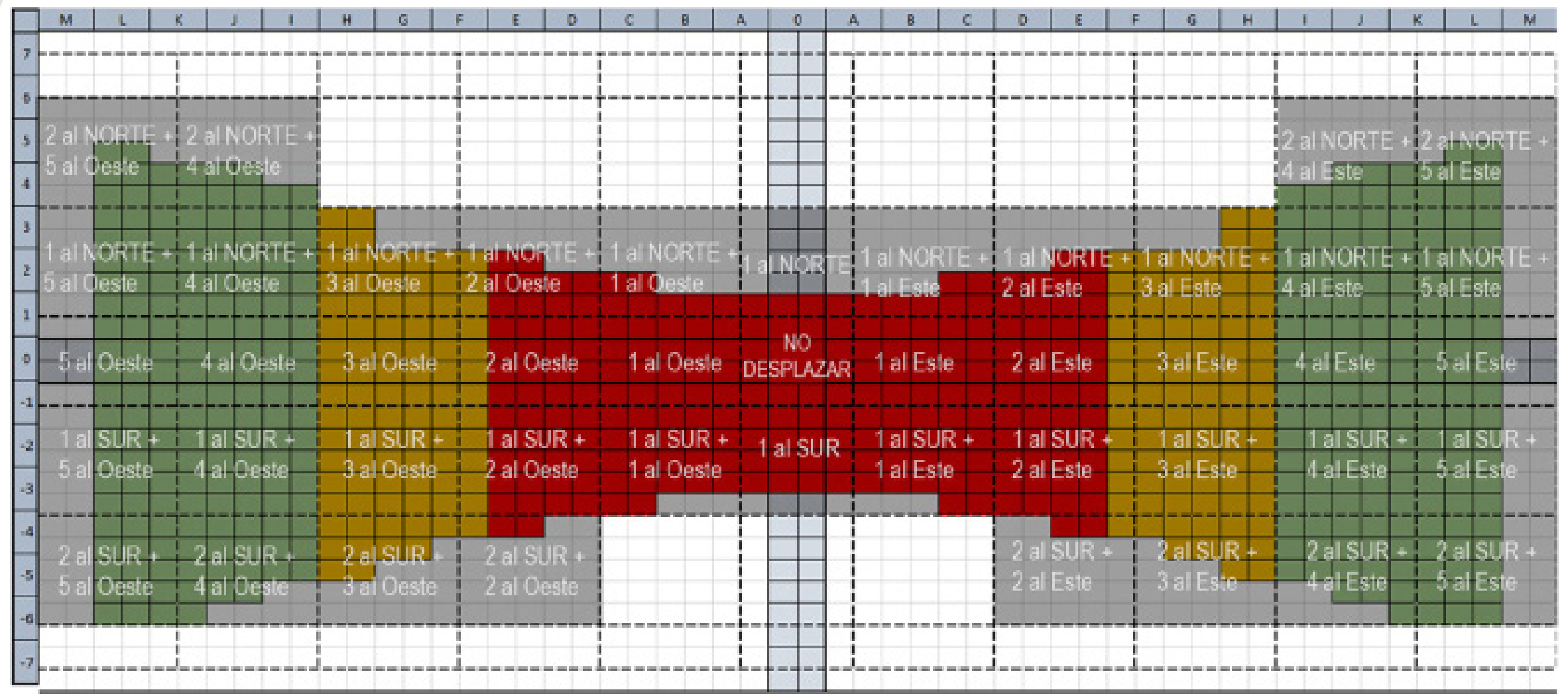

Figura 6. Sombreamiento ideal - Altura 6.0m. Fuente: elaboración propia.

Figura 7. Cuadricula de la malla espacial a diferentes alturas sobre una máscara solar. Fuente: elaboración propia.
El cálculo de las celdas resaltadas se hizo usando cálculos geométricos básicos de mecánica solar y apoyándose en máscaras solares (ver Figura 7).

\section{Ensayo de la herramienta}

A continuación, se muestra un ejemplo de aplicación de la herramienta previamente explicada. Como primera medida, se seleccionó el rango de tiempo en el que se desearía tener sombra sobre el punto central. En este caso fue seleccionado el rango entre 9:00h y 15:00h, periodo de mayor ganancia de calor por radiación solar directa. La Figura 8 muestra, en rojo, en qué celdas deberían disponerse los paneles horizontales a $5 \mathrm{~m}$ de altura para garantizar la sombra deseada en el punto 0,0 .

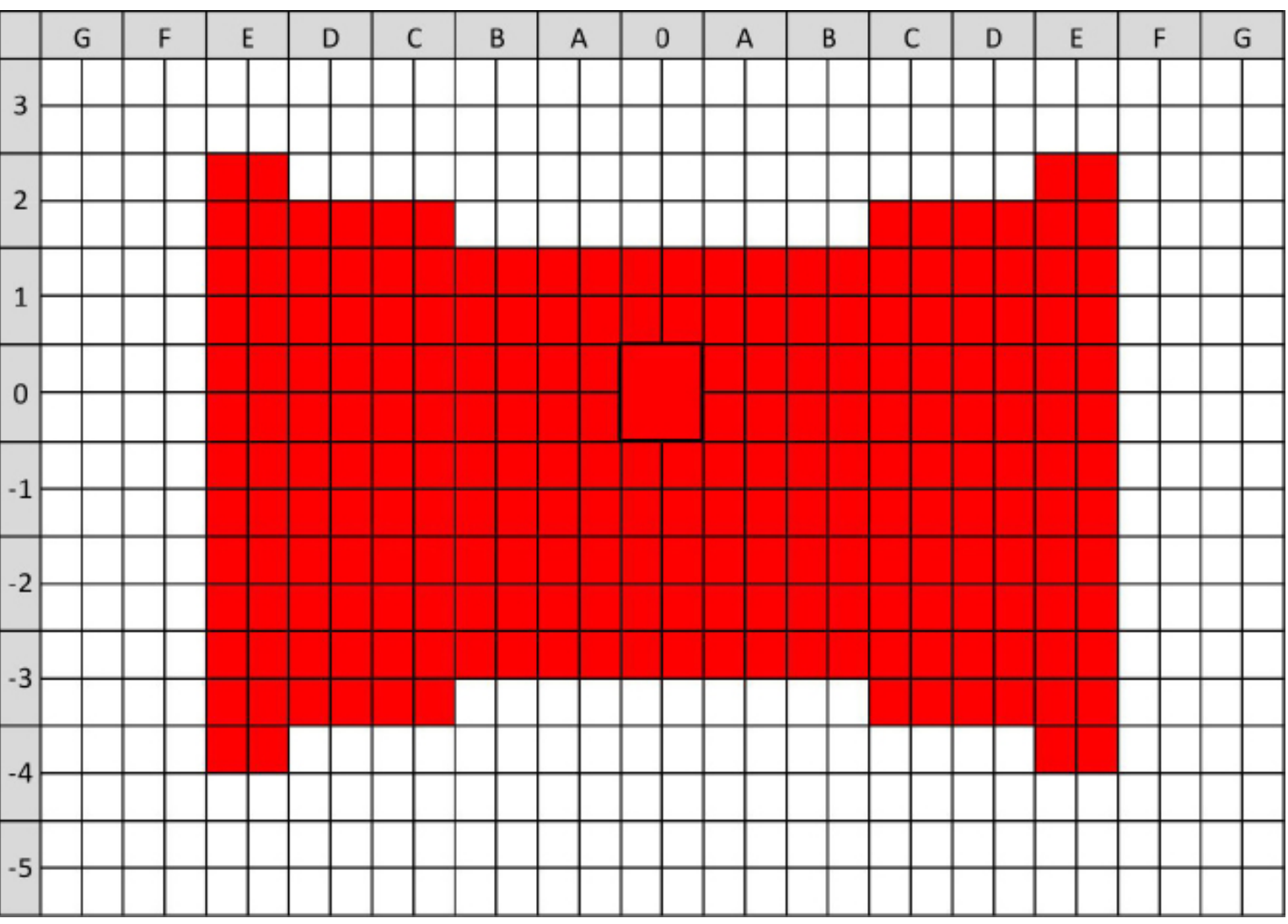

Sobre las celdas resaltadas en la figura anterior, el grupo de arquitectos diseñadores colocó un número 1, 2 ó 3 de manera aleatoria para indicar a que altura desearía disponer el panel que garantizaría la sombra sobre el rango de horas previamente indicado. En este caso, se usó 1 para paneles a $5 \mathrm{~m}$ de altura, 2 para paneles a 5,5m de altura y 3 para paneles a $6 \mathrm{~m}$ de altura. La Figura 9 ilustra un ejemplo de esta selección 
Figura 8. Obstrucción de celdas para garantizar protección solar a una altura de $5.0 \mathrm{~m}$ entre 9:00h y 15:00h.

Fuente: elaboración propia
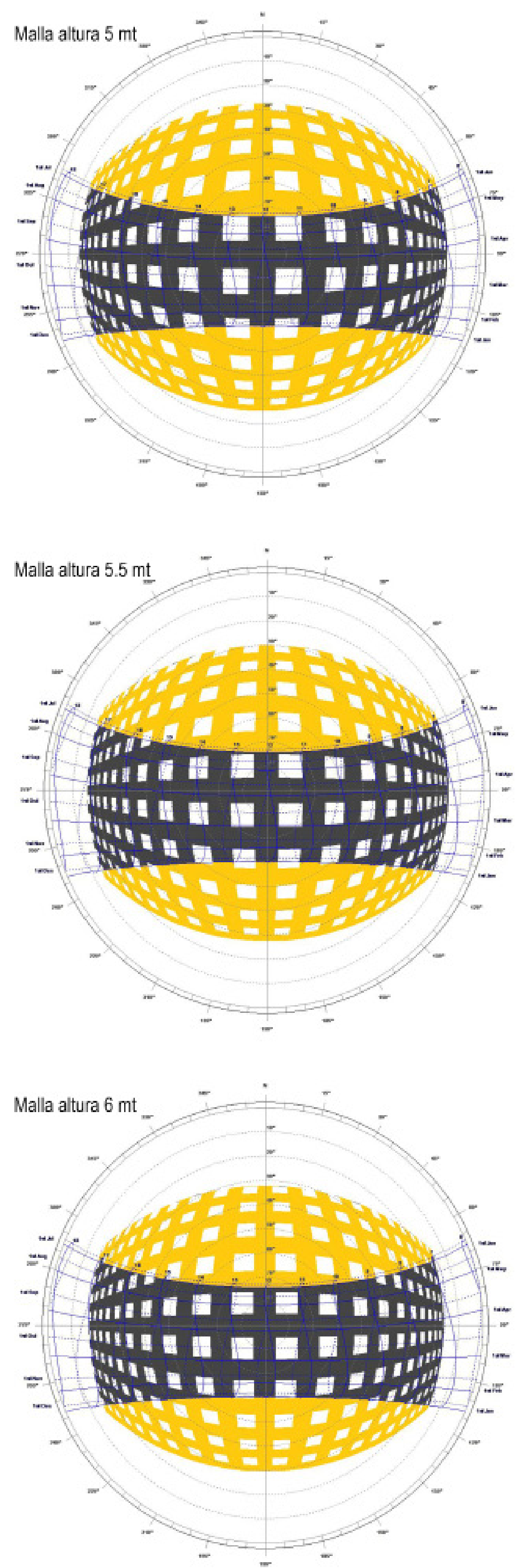


\begin{tabular}{|c|c|c|c|c|c|c|c|c|c|c|c|c|c|c|c|c|c|c|c|c|}
\hline & $G$ & $\mathrm{~F}$ & E & & D & $c$ & & $B$ & & & 0 & A & & & C & & & $E$ & F & $G$ \\
\hline & & & & & & & & & & & & & & & & & & & & \\
\hline & & & & & & & & & & & & & & & & & & & & \\
\hline & & & 2 & & & & & & & & & & & & & & & 1 & & \\
\hline 2 & & & 2 & & 3 & 3 & & & & & & & & & \begin{tabular}{l|l}
3 & 3 \\
\end{tabular} & 3 & & 2 & & \\
\hline & & & \begin{tabular}{|l|}
3 \\
\end{tabular} & & 1 & 1 & & & 2 & & 22 & & 1 & 1 & \begin{tabular}{l|l}
2 & 2
\end{tabular} & 3 & & 2 & & \\
\hline 1 & & & $3 \mid$ & & 1 & 1 & 1 & & 2 & & 22 & 35 & 1 & 1 & \begin{tabular}{l|l}
2 & 2
\end{tabular} & 1 & & 1 & & \\
\hline & & & \begin{tabular}{|l|}
3 \\
\end{tabular} & & 2 & 2 & 2 & 2 & & 1 & 11 & 11 & 3 & & 22 & & 1 & 11 & & \\
\hline & & & \begin{tabular}{|l|}
3 \\
\end{tabular} & & 3 & 2 & 2 & 2 & 1 & 1 & 11 & 11 & 3 & 3 & 22 & 3 & & 2 & & \\
\hline & & & 22 & & 3 & 2 & & $\sqrt{1}$ & & 3 & $3 \mid 3$ & 22 & 2 & 2 & 11 & 3 & & 22 & & \\
\hline & & & 22 & 21 & 1 & 2 & 2 & 1 & & 3 & $\begin{array}{l}33 \\
\end{array}$ & \begin{tabular}{|l|l}
2 & 2 \\
\end{tabular} & 2 & 2 & \begin{tabular}{l|l}
1 & 1
\end{tabular} & & 3 & 3 & & \\
\hline & & & 35 & & 1 & 3 & & 1 & & 2 & \begin{tabular}{l|l}
1 & 1
\end{tabular} & \begin{tabular}{|l|l}
1 & 1
\end{tabular} & 3 & & \begin{tabular}{l|l}
1 & 1
\end{tabular} & & 3 & 3 & & \\
\hline & & & 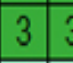 & & 1 & 3 & \begin{tabular}{|l|l}
3 & \\
\end{tabular} & 1 & 2 & \begin{tabular}{|l|}
2 \\
\end{tabular} & 11 & \begin{tabular}{|l|l}
1 & 1 \\
\end{tabular} & 3 & 3 & \begin{tabular}{l|l}
11 \\
\end{tabular} & 3 & & 2 & & \\
\hline & & & 11 & 12 & 2 & 2 & & 1 & & 3 & 32 & & 3 & 3 & \begin{tabular}{|l|}
3 \\
\end{tabular} & 2 & 2 & 2 & & \\
\hline & & & \begin{tabular}{l|l|}
1 & 1
\end{tabular} & & & & & & & & & & & & 3 & & 2 & 21 & & \\
\hline & & & 11 & & & & & & & & & & & & & & & 3 & & \\
\hline & & & & & & & & & & & & & & & & & & & & \\
\hline & & & & & & & & & & & & & & & & & & & & \\
\hline & & & & & & & & & & & & & & & & & & & & \\
\hline
\end{tabular}

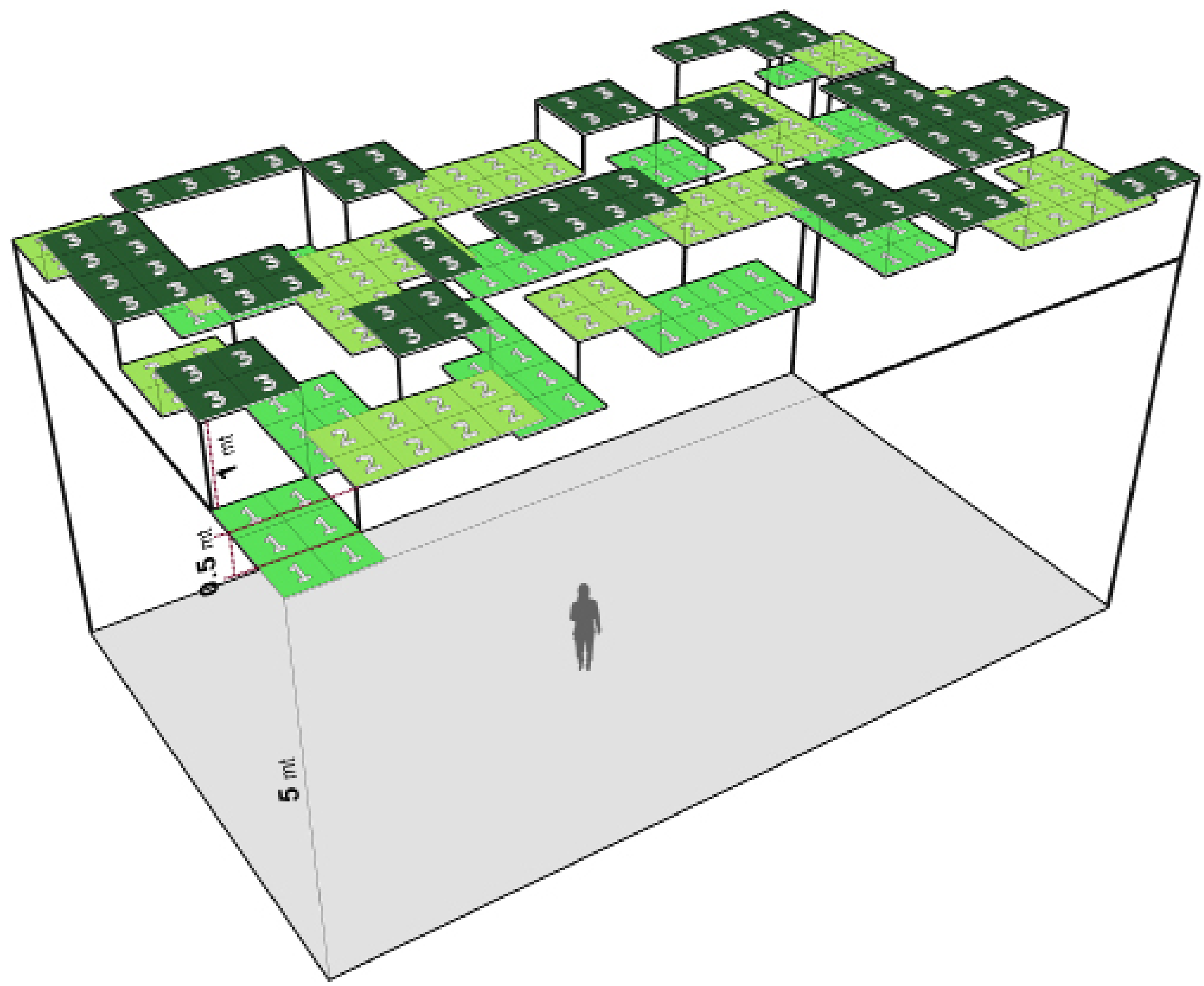

Figura 9. Selección de altura del panel para

garantizar sombre deseada.

Fuente: elaboración propia. 
Figura 10. Celdas con paneles para sombrear a > $5.0 \mathrm{~m}$ de altura.

Fuente: elaboración propia.
Paso seguido, se realizó la primera depuración. Eliminando los números 2 y 3 de la Figura 9, quedaron las celdas resaltadas con el número 1, que corresponden a la sombra generada por los dispositivos a $5 \mathrm{~m}$ de altura. Debido a que la sombra ideal de la Figura 8 también se encuentra a $5 \mathrm{~m}$ de altura, la sombra generada corresponde al panel dispuesto a esa misma altura. Por tanto, los paneles de $5 \mathrm{~m}$ estarían dispuestos en la misma ubicación marcada preliminarmente.

\begin{tabular}{|c|c|c|c|c|c|c|c|c|c|c|c|c|c|c|c|c|c|c|c|c|c|c|c|c|}
\hline & G & $F$ & 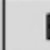 & 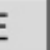 & D & & C & & B & & A & 0 & & A & & B & & C & D & & $\mathrm{E}$ & & $\mathrm{F}$ & G \\
\hline 3 & & & & & & & & & & & & & & & & & & & & & & & & \\
\hline & & & & & & & & & & & & & & & & & & & & & & & & \\
\hline 2 & & & & & & & & & & & & & & & & & & & & & 13 & & & \\
\hline 2 & & & & & & & & & & & & & & & & & & & & & & & & \\
\hline 1 & & & & & 1 & 1 & 1 & 1 & & & & & & & & 1 & 1 & & & & & & & \\
\hline 1 & & & & & 1 & 1 & 1 & 1 & & & & & & & & 1 & 1 & & 1 & 1 & 11 & 1 & & \\
\hline 0 & & & & & & & & & & & 11 & 1 & 1 & 1 & 1 & & & & 1 & 1 & 11 & 1 & & \\
\hline 0 & & & & & & & & & & & \begin{tabular}{l|l}
1 & 1
\end{tabular} & 1 & 1 & 1 & 1 & & & & & & & & & \\
\hline-1 & & & & & & & & & & 1 & & & & & & & & \begin{tabular}{l|l}
1 & 1 \\
\end{tabular} & & & & & & \\
\hline 1 & & & & & 1 & 1 & & & & 1 & & 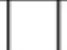 & & 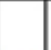 & 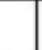 & & . & $\begin{array}{lll}1 & 1\end{array}$ & & & & & & \\
\hline-2 & & & & & 1 & 1 & & & 1 & 1 & & 1 & 1 & 1 & 1 & & & \begin{tabular}{l|l}
1 \\
1
\end{tabular} & & & & & & \\
\hline & & & & & 1 & 1 & & & 1 & 1 & & 1 & 1 & 1 & 1 & & 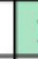 & $\begin{array}{lll}1 & 1\end{array}$ & & & & & & \\
\hline-3 & & & 1 & 1 & & & & & 1 & 1 & & & & & & & & & & & & & & \\
\hline & & & 1 & 1 & & & & & & & & & & & & & & & & & & & & \\
\hline-4 & & & 1 & 1 & & & & & & & & & & & & & & & & & & & & \\
\hline & & & & & & & & & & & & & & & & & & & & & & & & \\
\hline 5 & & & & & & & & & & & & & & & & & & & & & & & & \\
\hline & & & & & & & & & & & & & & & & & & & & & & & & \\
\hline
\end{tabular}

Posteriormente, se realizó la segunda depuración. De la Figura 9 se aislaron las celdas con número 2, que obedecen a paneles de $5.5 \mathrm{~m}$ de altura, teniendo como resultado la Figura 11. No obstante, tal como fue explicado, las celdas marcadas corresponden a la sombra que deberían generar los dispositivos localizados a 5,5m para satisfacer las necesidades de sombra. Por tanto, se aplica un factor de corrección de la localización de los dispositivos tal como se describió en la Figura 5. A continuación, la Figura 12 ilustra la localización de los paneles una vez realizado el ajuste.

\begin{tabular}{|c|c|c|c|c|c|c|c|c|c|c|c|c|c|c|c|c|c|c|c|c|c|c|c|c|}
\hline & G & $F$ & & $E$ & D & & C & & B & & A & & 0 & 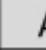 & $A$ & B & & C & & & $\mathrm{E}$ & & $F$ & G \\
\hline \multirow{2}{*}{\multicolumn{25}{|c|}{3}} \\
\hline & & & & & & & & & & & & & & & & & & & & & & & & \\
\hline \multirow{2}{*}{2} & & & 2 & 2 & & & & & & & & & & & & & & & & & & & & \\
\hline & & & 2 & 2 & & & & & & & & & & & & & & & & & 2 & 2 & & \\
\hline \multirow{2}{*}{1} & & & & & & & & & & 2 & 22 & 2 & 2 & & & & & $\begin{array}{ll}22 \\
\end{array}$ & & & 2 & 2 & & \\
\hline & & & & & & & & & & i & 2 & 2 & 2 & & & & & $\begin{array}{ll}2 & 2\end{array}$ & & & & & & \\
\hline \multirow{2}{*}{0} & & & & & 2 & 2 & 2 & 2 & 2 & 2 & & & & & & & & $\begin{array}{ll}2 & 2\end{array}$ & & & 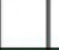 & 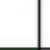 & & \\
\hline & & & & & & & 2 & 2 & 2 & 2 & & & & & & & & 22 & & & 2 & 2 & & \\
\hline \multirow{2}{*}{-1} & & & 2 & 2 & & & 2 & 2 & & & & & & 2 & 2 & 2 & 2 & & & & 2 & 2 & & \\
\hline & & & 2 & 2 & & & 2 & 2 & & & & & & 2 & 2 & 2 & 2 & & & & & & & \\
\hline \multirow{2}{*}{-2} & & & & & & & & & & 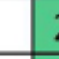 & 2 & & & & & & & & & & 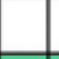 & . & & \\
\hline & & & & & & & & & & 2 & 2 & & & & & & & & & & 2 & 2 & & \\
\hline \multirow{2}{*}{-3} & & & & & 2 & 2 & 2 & 2 & & & & & 2 & 2 & 2 & & & & 2 & 2 & 2 & 2 & & \\
\hline & & & & & 2 & 2 & 2 & 2 & & & & & & & & & & & 2 & 2 & 2 & 2 & & \\
\hline \multirow{2}{*}{-4} & & & & & & & & & & & & & & & & & & & & & & & & \\
\hline & & & & & & & & & & & & & & & & & & & & & & & & \\
\hline & & & & & & & & & & & & & & & & & & & & & & & & \\
\hline & & & & & & & & & & & & & & & & & & & & & & & & \\
\hline
\end{tabular}

Figura 11. Celdas con paneles para sombrear a $5.5 \mathrm{~m}$ de altura.

Fuente: elaboración propia. 
Figura 12. Corrección de localización de paneles a $5.5 \mathrm{~m}$ de altura.

Fuente: elaboración propia.

Figura 13. Celdas con paneles para sombrear a > $6.0 \mathrm{~m}$ de altura.

Fuente: elaboración propia.

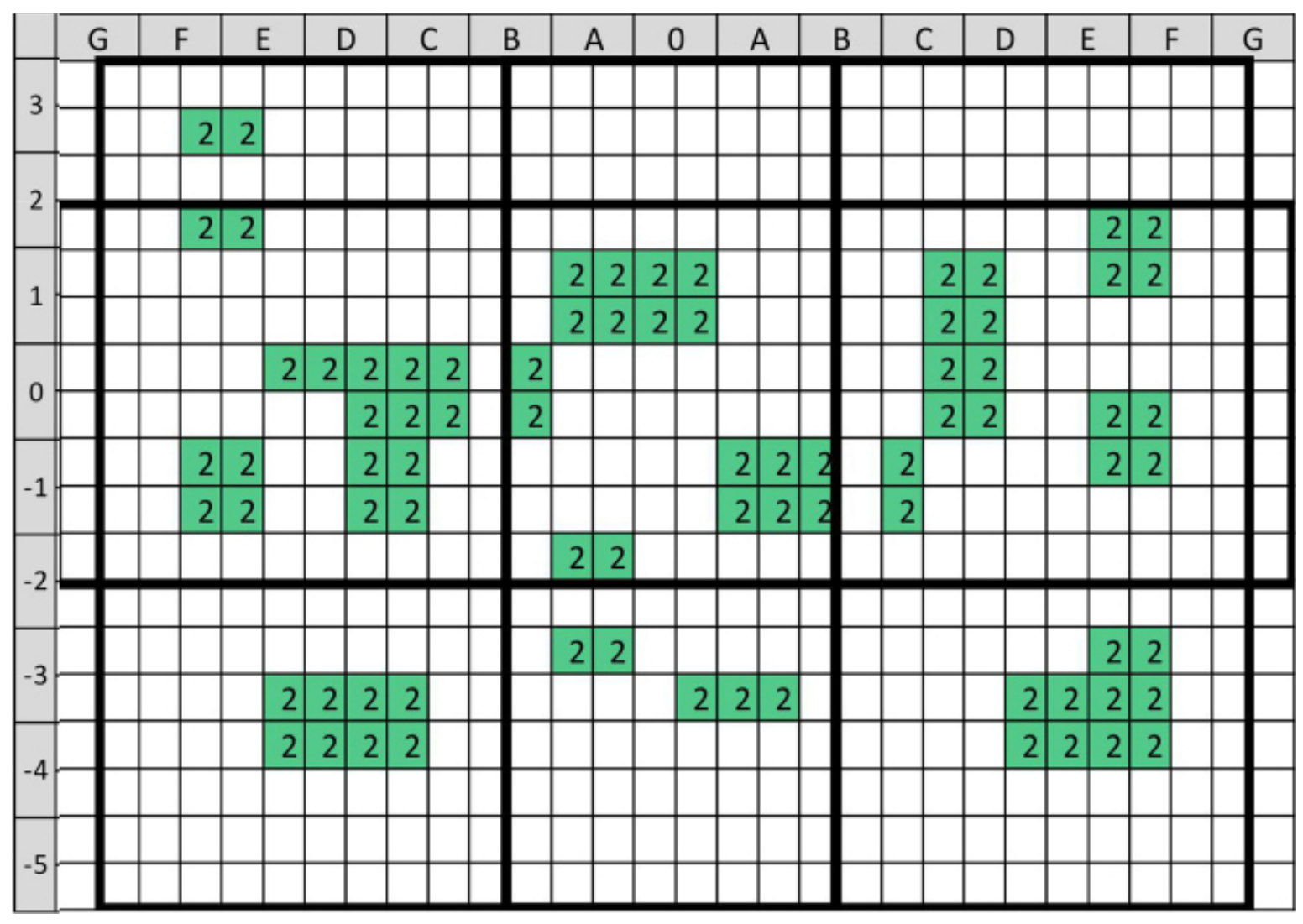

Similar a lo realizado con la cubierta a $5,5 \mathrm{~m}$, pero ya haciendo referencia a la altura $6 \mathrm{~m}$, se aislaron los números tres (3) de la Figura 9, tal como lo muestra la Figura 13.

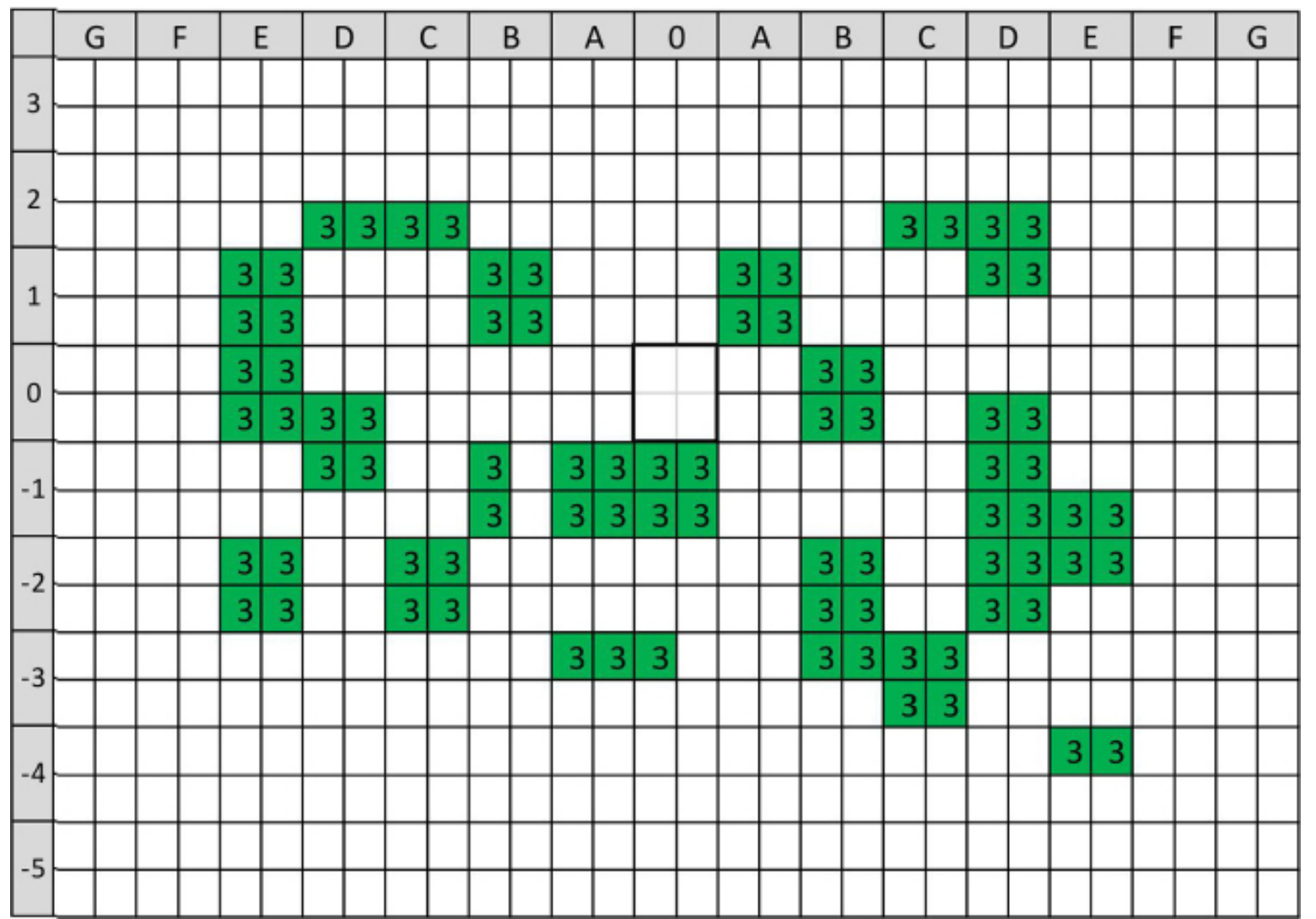

Finalmente, a fin de calcular la posición de los paneles a $6 \mathrm{~m}$ que generarían las sombras en el rango deseable, se aplicaron las correcciones de localización tal como se referenció en la explicación del método. La Figura 14 muestra la localización de los paneles o dispositivos de sombra a $6 \mathrm{~m}$ de altura.

Los factores de corrección permiten reacomodar la posición de los dispositivos de sombra de acuerdo con su altura, redefiniendo geométricamente la propuesta de cubierta y garantizando sombra en los momentos deseados. De esta, el grupo de arquitectos pudo definir la disposición de los paneles considerando criterios para sombrear según los periodos de tiempo predefinidos. La Figura 15 muestra el resultado final de la disposición de elementos. El resultado muestra en colores las horas-día promedio de exposición a la radiación solar directa. En la parte central o punto para el cual fue diseñada la disposición de elemento de sombra, se evidencia menos de 3 horas de exposición media diaria considerando cielo despejado permanente. Debido a la aplicación de los factores de corrección, podría generase errores en la disposición de los dispositivos de sombra para proteger del sol de medio día. Por esta razón, debe prestarse especial atención a este rango de horarios en el resultado final. 
Figura 14. Corrección de localización de paneles > a $6.0 \mathrm{~m}$ de altura.

Fuente: elaboración propia.

Figura 15a. Disposición final de paneles para generar sombra a diferentes alturas /

simulaciones de exposición solar.

Fuente: elaboración propia.

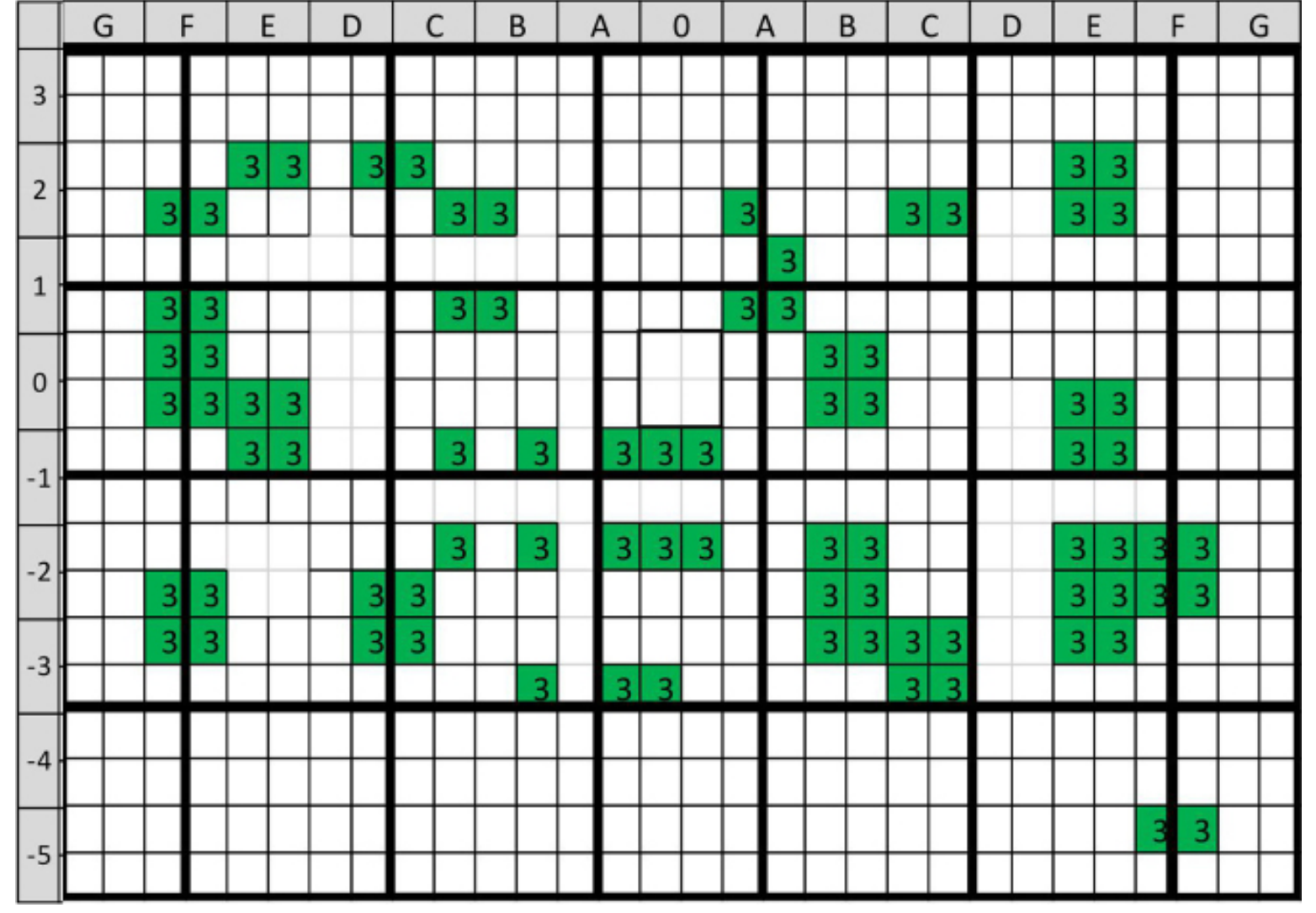

\begin{tabular}{|c|c|c|c|c|c|c|c|c|c|c|c|c|c|c|c|c|c|c|c|c|c|c|c|c|c|c|c|}
\hline & $G$ & f & & $\mathrm{E}$ & & D & & $c$ & & B & & A & & 0 & & A & & B & & & D & & $\mathrm{E}$ & & $f$ & & G \\
\hline \multirow[b]{2}{*}{3} & & & & & & & & & & & & & & & & & & & & & & & & & & & \\
\hline & & & 2 & 2 & & & & & & & & & & & & & & & & & & & & & & & \\
\hline \multirow{2}{*}{2} & & & & 3 & $\frac{3}{3}$ & & j: & 3 & & & & & & & & & & & & & & & & 3 & 1 & & \\
\hline & & 3 & 3 & 2 & & & & - & 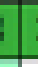 & 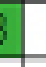 & & & & & $=$ & 3 & & & 3 & 3 & & & $\frac{3}{3}$ & 1 & 2 & & \\
\hline \multirow{2}{*}{1} & & & & & & 1 & 1 & 1 & 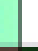 & & & 22 & 22 & 2. & 2 & 3 & 1 & 1 & & 2 & 2 & & & 2 & 2 & & \\
\hline & & 3 & 3 & & & I & 1 & 1 & 1 & 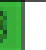 & & 27 & 27 & 27 & 2 & 33 & $t$ & 11 & & 2 & 2 & 1 & i & 1 & & & \\
\hline \multirow{3}{*}{0} & & 3 & 3 & & 2 & 2 & 2 & 2 & 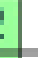 & 2 & 2 & 1 & 1 & 1 & 11 & \begin{tabular}{l|l}
1 & 1
\end{tabular} & 1 & 3 & & 2 & 2 & 11 & 1 & 1 & . & & \\
\hline & & 3 & 3 & 3 & 3 & & 2 & 2 & & $z$ & 21 & 11 & 1 & 1 & 15 & 11 & 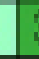 & 13 & & 2 & 2 & & 4 & 4 & 2 & & \\
\hline & & & 2 & 3 & 3 & & 2 & 2 & & 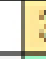 & 3 & 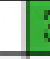 & 3 & 3 & 3 & 23 & & & 2 & 1 & & & 3 & 3 & 2 & & \\
\hline-1 & & & 2 & 2 & & 1 & 2 & 2 & & 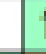 & | & . & 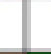 & ( & 1 & 22 & 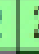 & & 2 & 1 & & & . & ( & 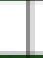 & & \\
\hline \multirow[b]{2}{*}{2} & & & & & & 1 & 1 & 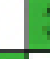 & 1 & 13 & 32 & 2 & 3 & 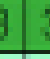 & 3 & & 4 & 3 & 1 & 1 & & & 1 & 3 & 3 & 3 & \\
\hline & & 3 & 3 & & & 1 & 3 & 3 & & 1 & 1 & & 1 & 1 & 1 & 11 & & 33 & 1 & 1 & & & 3 & 3 & 3 & 3 & \\
\hline \multirow{2}{*}{3} & & 3 & 3 & 1 & 1 & & 3 & 3 & & 11 & 12 & $2:$ & 2 & 1 & 18 & 10 & & 13 & 3 & 3 & & & 1 & 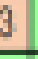 & 2 & & \\
\hline & & & & 1 & 2 & 2 & 2 & 2 & & & $\frac{3}{3}$ & & 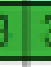 & 3 & 23 & 23 & & & 3 & 3 & & 2 & 2 & 2 & 2 & & \\
\hline & & & & 1 & 2 & 2 & 2 & 2 & & & & & & & & & & & & & & 2 & 2 & 2 & 2 & & \\
\hline & & & & & & & & & & & & & & & & & & & & & & & & & & & \\
\hline & & & & & & & & & & & & & & & & & & & & & & & & & $\frac{3}{3}$ & 3 & \\
\hline & & & & & & & & & & & & & & & & & & & & & & & & & & & \\
\hline
\end{tabular}

Itersocinom 1

Itaresponon 2

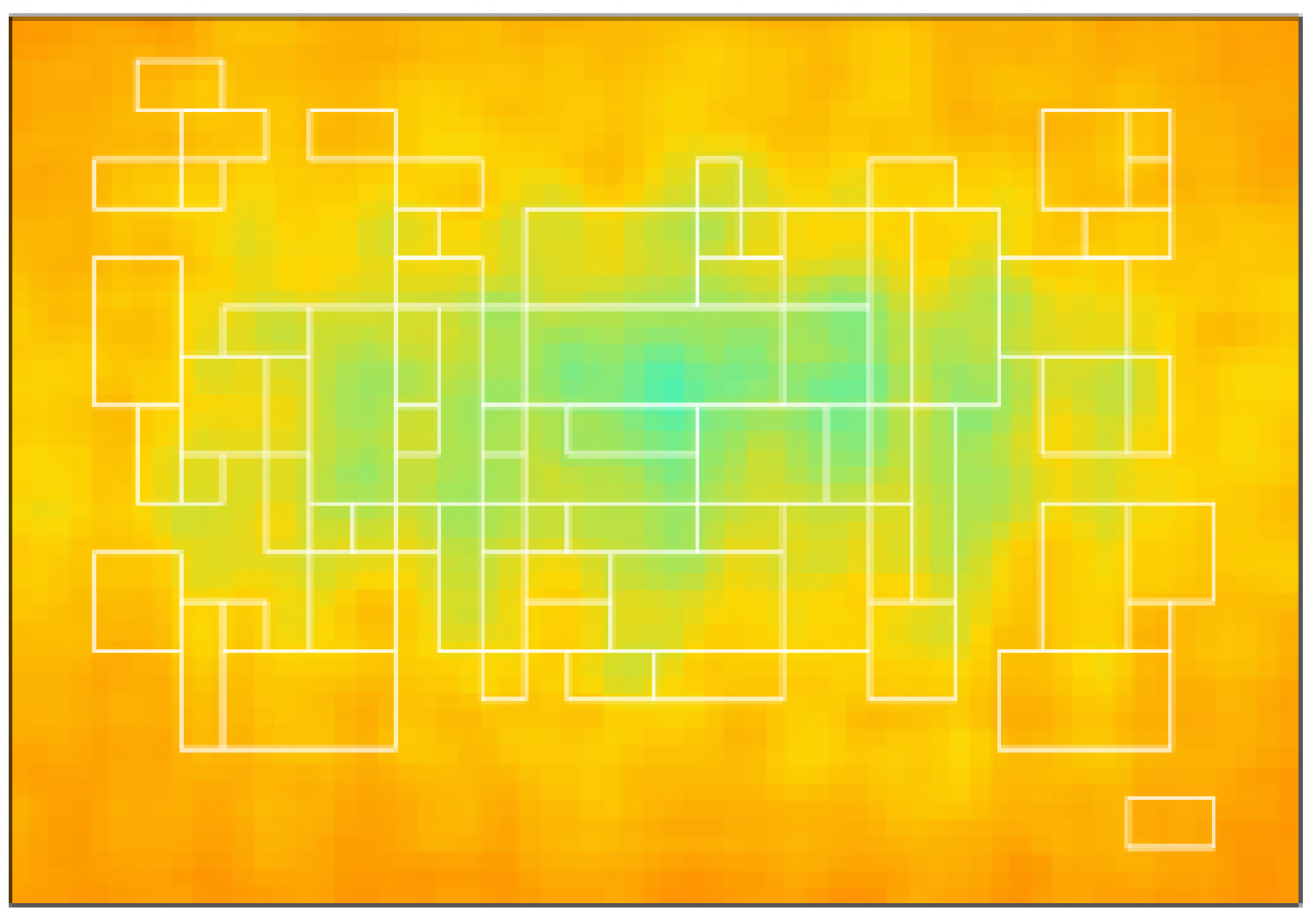




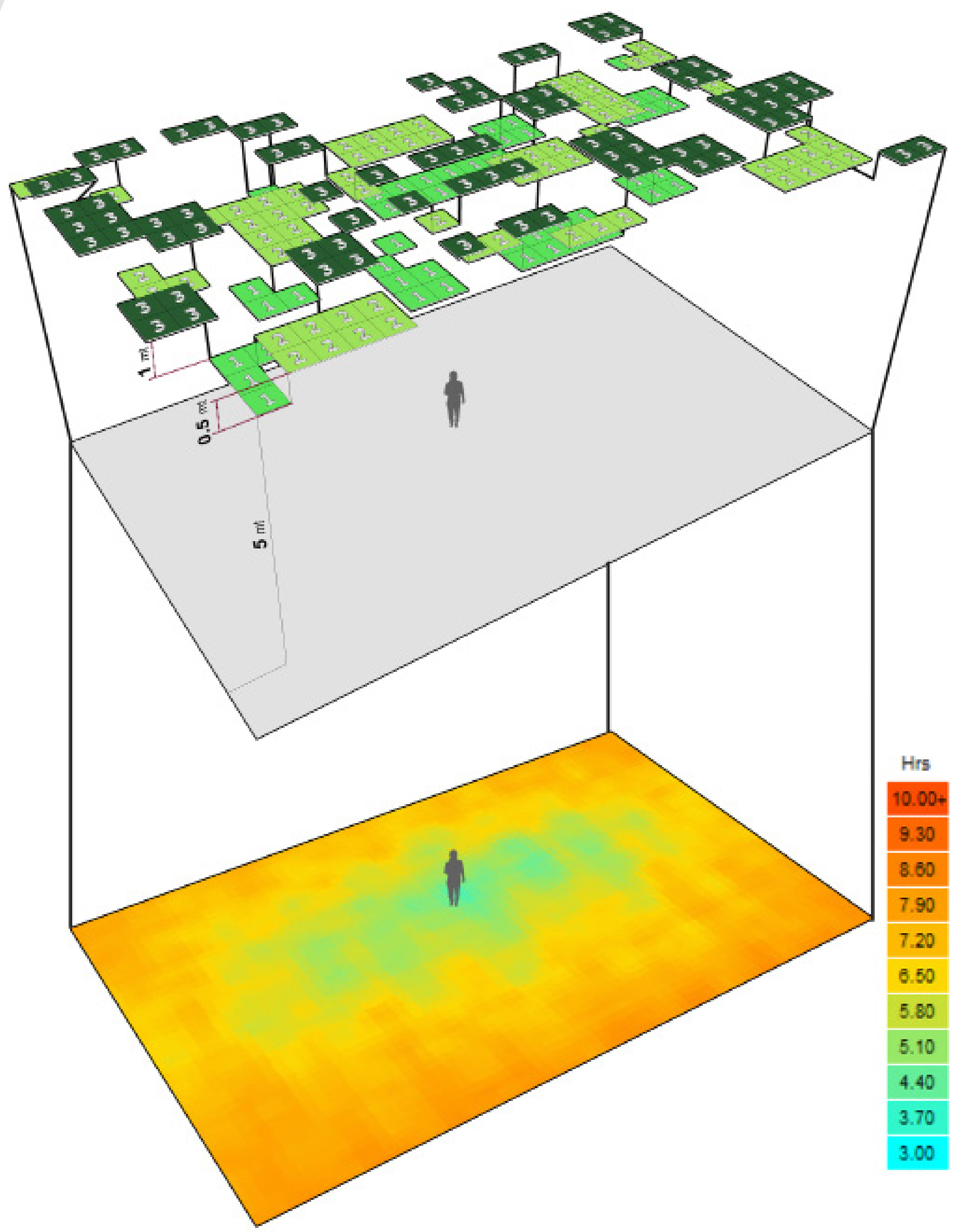

Figura 15b. Disposición final de paneles

para generar sombra a diferentes alturas /

A

simulaciones de exposición solar.

Fuente: elaboración propia. 
La Figura 16 muestra modelos tridimensionales de la propuesta final del grupo de arquitectos.

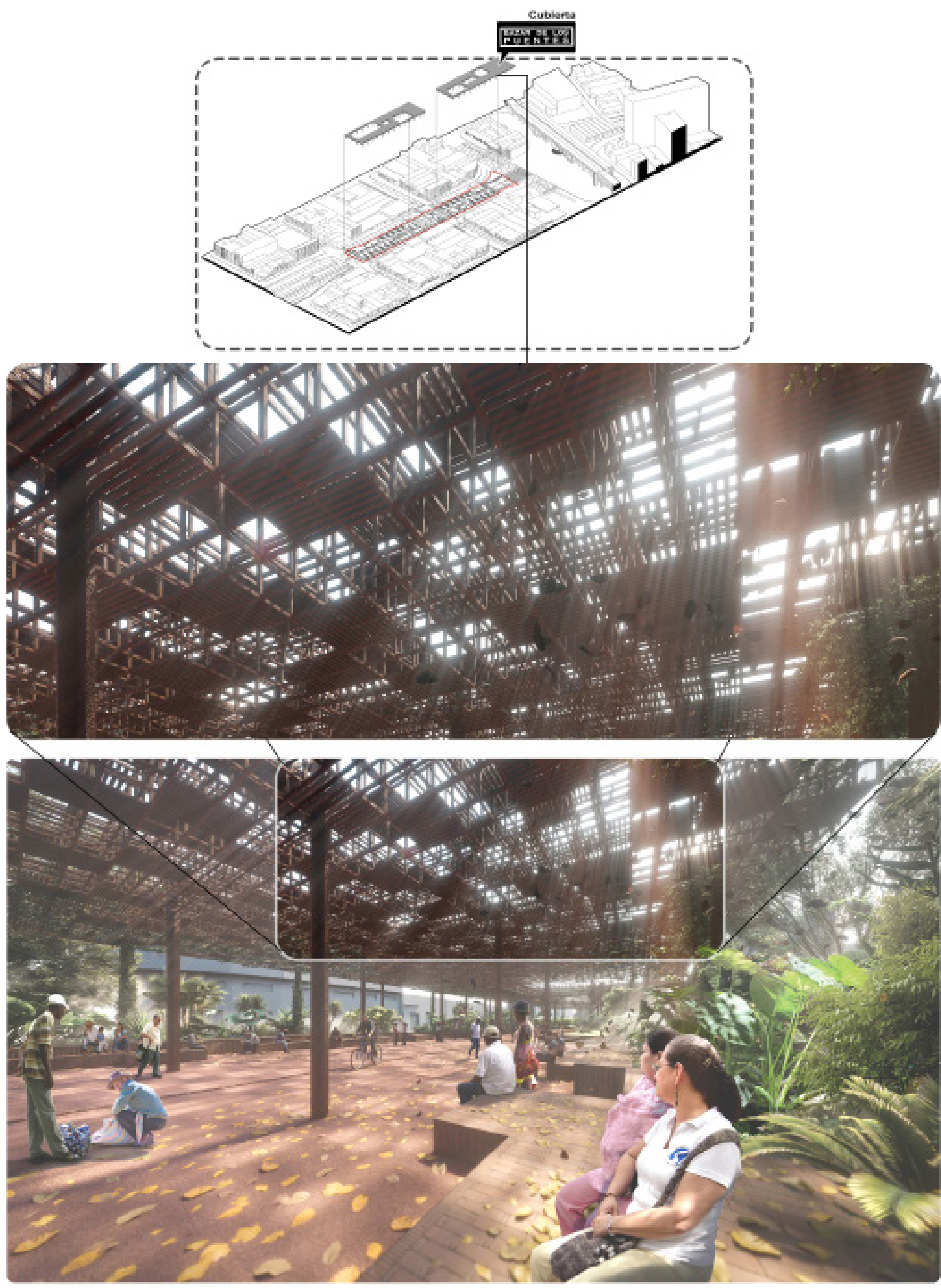




\section{Conclusiones}

El diseño de cubiertas en espacios exteriores a partir de un método tipo ensayo error, en el que se evalúen y valoren diversas posibilidades de configuración de los elementos que generan sombra, podría ser un proceso altamente tedioso y que requiera una considerable inversión de tiempo. Además de esto, dependiendo de la etapa en la que se encuentre el proceso proyectual, el ejercicio bioclimático a través de ese método podría arrojar resultados imposibles de lograr por temas de presupuesto o de cumplimiento de cronogramas.

Ante este panorama, la implementación de métodos proyectuales que posibiliten el diseño y disposición de elementos de sombra de acuerdo con criterios de protección solar previamente definidos puede garantizar que las intenciones estéticas, formales 0 de lenguaje del proyecto se alineen con las estrategias para garantizar comodidad térmica. Además, si el método se puede implementar desde las fases preliminares del proceso proyectual, la posibilidad de minimizar reprocesos en las partes finales aumenta.

La herramienta aplicada en el proceso de diseño del proyecto Bazar de los Puentes permitió que los arquitectos diseñadores definieran la disposición de paneles para sombrear casi como un juego, en el que de manera aleatoria propusieron la altura a la que querían generar la sombra durante un periodo de tiempo para un punto en el espacio, y permitió que el grupo de asesores de bioclimática se dedicara a validar y refinar los resultados del ejercicio. Esta dinámica representa un avance altamente valioso, pues permite efectivamente integrar la bioclimática al proceso proyectual, no como un proceso anexo, sino como el proceso mismo de diseño en el que la bioclimática se vuelve el eje rector del proceso proyectual.

\section{Referencias bibliográficas}

Ahmed, K. S. (2003). Comfort in urban spaces: Defining the boundaries of outdoor thermal comfort for the tropical urban environments. Energy and Buildings, 35(1), 103-110. https://doi.org/10.1016/S0378-7788(02)00085-3

Arango-Díaz, L., Giraldo Vásquez, N., Rupp, R., Sierra, J., \& Gallego, F. (2015). Sombreamiento de ambientes urbanos en la ladera oriental de medellín, Colombia. Caso de estudio: Parque ecoturístico y Recreativo de Golondrinas. In XIII Encontro Nacional e IX Encontro Latino-americano de Conforto no Ambiente Construído (pp. 3-10). Campinas.

Aroztegui, J. M. (1980). Método para projeto e avaliacao de pára-sois externos, visando á ptimizacaio de seu desempenho tpermico como unm clima dado.

Bittencourt, L. (1988). Uso das cartas solares: diretrizes para arquitetos. Universidade Federal de Alagoas.

Boukhelkhal, I., \& Bourbia, P. F. (2016). Thermal comfort conditions in outdoor urban spaces: Hot dry climate -Ghardaia- Algeria. Procedia Engineering, 169, 207-215. https://doi.org/10.1016/j.proeng.2016.10.025

Cheng, B., Gou, Z., Zhang, F., Feng, Q., \& Huang, Z. (2019). Thermal comfort in urban mountain parks in the hot summer and cold winter climate. Sustainable Cities and Society, 51(August), 101756. https://doi.org/10.1016/j.scs.2019.101756

Dubois, M. (2000). Proceedings of the Third ISES Europe Solar Congress: Eurosun 2000, 19-22, (1957), 19-22.

Dutra, L., Lamberts, R., Oscar, F., \& Pereira, R. (s.f.). Determinação do fator solar desejável em aberturas Térmica Luciano Dutra, Roberto Lamberts, Fernando Oscar Rutkay Pereira conforto térmico, energia, proteções solares, edificações.

García, A., \& Salazar, J. H. (2006). De lo cotidiano a lo científico. Las maneras pedagógicas de la vida cotidiana en la enseñanza de la tecnología en arquitectura. Revista Educación y Pedagogía, XVIII, 87-92. 
González, D., \& Martínez, R. (2014). Sistema de elementos de protección solar para los edificios en Cuba. Estudio de caso. Arquitectura y Urbanismo, XXXV(3), 5-17.

Haghparast, F., \& Marsh, A. (2004). The Application of computer-otimised solutions to tightly definded design problems. In Plea 2004. Eindhoven.

Kaftan, E. (2001). The cellular method to design energy efficient shading form to accommodate the dynamic characteristics of climate. Plea, 829-833.

Kaftan, E., \& Marsh, A. (2005). Integrating the cellular method for shading design with a thermal simulation. Passive and Low Energy Cooling for the Built Environment, (May), 965-970.

Kicovic, D., Vuckovic, D., Markovic, D., \& Jovic, S. (2019). Assessment of visitors' thermal comfort based on physiologically equivalent temperature in open urban areas. Urban Climate, 28(January), 100-466. https://doi.org/10.1016/j. uclim.2019.100466

Lai, D., Liu, W., Gan, T., Liu, K., \& Chen, Q. (2019). A review of mitigating strategies to improve the thermal environment and thermal comfort in urban outdoor spaces. Science of the Total Environment, 661, 337-353. https://doi.org/10.1016/j. scitotenv.2019.01.062

Lin, T. P., Matzarakis, A., \& Hwang, R. L. (2010). Shading effect on long-term outdoor thermal comfort. Building and Environment, 45(1), 213-221. https://doi. org/10.1016/j.buildenv.2009.06.002

Marsh, A., \& Carruthers, D. (1995). A selection of Interactive Design Tools. ANZAScA Conference, (June).

Marsh, A. J. (1996). Integrating performance modelling into the initial stages of design. ANZAScA Conference Proceedings.

Mazhar, N., Brown, R. D., Kenny, N., \& Lenzholzer, S. (2015). Thermal comfort of outdoor spaces in Lahore, Pakistan: Lessons for bioclimatic urban design in the context of global climate change. Landscape and Urban Planning, 138, 110-117. https://doi.org/10.1016/j.landurbplan.2015.02.007

Mesa, N. A. y Morillón, D. (2005). Metodología para el diseño optimizado de las herramientas de control solar. Avances En Energías Renovables y Medio Ambiente, 9, 37-42.

Morillón Gálvez, D. y Mejía Domínguez, D. (2004). Modelo para diseño y evaluación del control solar en edificios.

Naranjo, A., Rupp, R. F., Arango-Díaz, L. A., Giraldo Vásquez, N., Sierra, J., Gallego, F., ... Galafassi, M. (2015). Proceso De Diseño Del Nuevo Centro Administrativo Del. En XIII Encontro Nacional e IX Encontro Latino-americano de Conforto no Ambiente Construído (pp. 1-9). Campinas.

Nikolopoulou, M., Baker, N., \& Steemers, K. (2001). Thermal comfort in outdoor urban spaces: Understanding the Human parameter. Solar Energy, 70(3), 227-235. https://doi.org/10.1016/S0038-092X(00)00093-1

Nikolopoulou, M., \& Steemers, K. (2003). Thermal comfort and psychological adaptation as a guide for designing urban spaces. Energy and Buildings, 35(1), 95-101. https://doi.org/10.1016/S0378-7788(02)00084-1

Ouali, K., El Harrouni, K., Abidi, M. L., \& Diab, Y. (2020). Analysis of Open Urban Design as a tool for pedestrian thermal comfort enhancement in Moroccan climate. Journal of Building Engineering, 28(October 2019), 101042. https://doi.org/10.1016/j. jobe.2019.101042

Salazar, J., García, A., González, A., Arango-Díaz, L., \& Giraldo, N. (2009). Criterios para el diseño de fachadas utilizando elementos prefabricados en las torres de oficinas. Proyecto Centro Cívico de Antioquia, Plaza de la Libertad, Medellín, Colombia. En $X$ encontro nacional e vi encontro latino americano de conforto no ambiente construido (pp. 1763-1772). 
Salazar, J. H. (2007). Sombra con elementos verticales. Diseño de las agrupaciones de las torres de luz de la plaza cisneros. Medellín, Colombia. IX Encontro Nacional V Latino Americano de Conforto No Ambiente Contruído ENCAC2007.

Salazar Trujillo, J. H. (2009). Sombra vegetal las huellas de sombra. ENCAC.

Salazar Trujillo, J. H. (2014). Calculation of the shadow-penumbra relation and its application on efficient architectural design. Solar Energy, 110, 139-150. https:// doi.org/10.1016/j.solener.2014.08.043

Salazar Trujillo, J. H., García Cardona, A. A., \& Gónzalez Castaño, A. (2006). Labor cero. Arquitectura a la medida. Colombia: ARGOS.

Torres-Gómez, A. M., Gallego-Cardona, H., Giraldo Vásquez, N., Arango-Díaz, L., Rupp, R. F., \& Naranjo-Gaviria, A. (2015). Ergonomia como ferramenta de projeto para configucaraco do ambiente construido: caso Unidade de Vida Articulada UVAOrfelinato. En 15 Ergodesign.

Villalba, A., Monteoliva, J., \& Pattini, A. (2011). Control solar sobre superficies vidriadas. Evaluación lumínica mediante métricas dinámicas y preferencia de usuarios a filtros solares. Avances En Energías Renovables y Medio Ambiente, 15(January 2015), 79-88.

Waldron, J. A. (2017). Human behaviour outdoors and the environmental factors. University of Nottingham.

Waldron, J. A., \& Salazar, J. H. (2013). Calculation of areas of permanence in public spaces, according to solar radiation simulated conditions. Lecture Notes in Computer Science (Including Subseries Lecture Notes in Artificial Intelligence and Lecture Notes in Bioinformatics), 8014 LNCS(PART 3), 215-223. https://doi. org/10.1007/978-3-642-39238-2_24

Wang, Y., Ni, Z., Peng, Y., \& Xia, B. (2018). Local variation of outdoor thermal comfort in different urban green spaces in Guangzhou, a subtropical city in South China. Urban Forestry and Urban Greening, 32(March), 99-112. https://doi.org/10.1016/j. ufug.2018.04.005

Xu, M., Hong, B., Jiang, R., An, L., \& Zhang, T. (2019). Outdoor thermal comfort of shaded spaces in an urban park in the cold region of China. Building and Environment, 155(January), 408-420. https://doi.org/10.1016/j.buildenv.2019.03.049

Yang, W., Wong, N. H., \& Jusuf, S. K. (2013). Thermal comfort in outdoor urban spaces in Singapore. Building and Environment, 59, 426-435. https://doi.org/10.1016/j. buildenv.2012.09.008

Zabetian, E., \& Kheyroddin, R. (2019). Comparative evaluation of relationship between psychological adaptations in order to reach thermal comfort and sense of place in urban spaces. Urban Climate, 29(January), 100-483. https://doi.org/10.1016/j. uclim.2019.100483 